\title{
FORÇA DE PREENSÃO PALMAR E FREQUENCIA CARDÍACA EM ATLETAS AMADORAS DE HANDEBOL ${ }^{1}$
}

\author{
HANDGRIP STRENGHT AND HEART RATE IN \\ AMATEUR HANDBALL ATHLETES
}

\section{Deborah Paula Almeida Zaltron ${ }^{2}$, Lilian Oliveira de Oliveira ${ }^{3}$, João Rafael Sauzem Machado ${ }^{4}$, Minéia Weber Blattes ${ }^{5}$, Luiz Fernando Rodrigues Junior ${ }^{6}$ e Jaqueline de Fátima Biazus ${ }^{7}$}

\section{RESUMO}

O handebol é definido como um esporte olímpico de alta intensidade, pois exige demandas aeróbicas e de força muscular esquelética. Contém elementos táticos de grande importância, como ações de corrida, arremesso, salto, contato e bloqueio. Essas atividades acarretam frequência cardíaca média de treinamento acima de 80\% da frequência cardíaca máxima. A composição corporal também é importante fator que pode influenciar no desempenho do jogador em uma partida, tendo em vista que o tamanho da mão e a força de preensão de cada jogador têm influência direta no controle da bola. No presente estudo, teve-se como objetivo avaliar a força de preensão e a frequência cardíaca de treinamento em atletas amadoras de handebol. Participaram deste estudo 12 atletas com idade média de 19,8 anos. A força de preensão média foi de 5,4 $\pm 1,2 \mathrm{kgf}$ no MSD e 5,3 $\pm 0,85 \mathrm{kgf}$ em MSE. A frequência cardíaca em treinamento foi de 79\% FCmáx. Concluiu-se que não há diferença estatística em relação à força de preensão entre os membros superiores das atletas e que existe uma homogeneidade da frequência cardíaca das atletas ao longo do treinamento.

Palavras-chave: dinamômetro de força muscular, membro superior, handebol.

\section{ABSTRACT}

Handball is defined as a high intensity Olympic sport, requiring aerobic demands and skeletal muscle strength, containing tactical elements of great importance such as running, throwing, jumping, contact and blocking actions. These activities lead to an average training heart rate above $80 \%$ of the maximum heart rate. Body composition is also an important factor that can influence the player's performance in a match, given that the size of the hand and the grip strength of each player has a direct influence on the control of the ball. The present study aimed to evaluate the grip strength and the training heart rate from amateur handball athletes. Twelve athletes with an average age of 19.8 years participated in this study. The grip strength average was $5.4 \pm 1.2 \mathrm{~kg} f$ to RUL (right upper limb) and $5.3 \pm 0.85 \mathrm{~kg} f$ to LUL (left upper limb). The training heart rate average was $79 \%$ FCmax. It is concluded that there is no statistical difference in relation to the grip strength between the upper limbs of the athletes and that there is a homogeneity in the heart rate of the athletes throughout the training.

Keywords: muscle strength dynamometer, upper limb, handball.

\footnotetext{
${ }^{1}$ Trabalho final de graduação.

${ }^{2}$ Acadêmica do Curso de Fisioterapia - Universidade Franciscana - UFN. E-mail: deborahzaltron8@gmail.com

${ }^{3}$ Colaboradora. Docente do Curso de Fisioterapia - Universidade Franciscana - UFN. E-mail: licafisiot@hotmail.com

${ }^{4}$ Colaborador. Docente do Curso de Fisioterapia - Universidade Franciscana - UFN. E-mail: drjoaorafa@yahoo.com.br

${ }^{5}$ Colaboradora. Docente do Curso de Farmácia - Universidade Franciscana - UFN. E-mail: mineia_weber@yahoo.com.br

${ }^{6}$ Colaborador. Docente do Curso de Engenharia Biomédica - Universidade Franciscana - UFN. E-mail: luiz.fernando@ ufn.edu.br

${ }^{7}$ Orientadora. Docente do Curso de Fisioterapia - Universidade Franciscana - UFN. E-mail: jaquebiazus@hotmail.com
} 


\section{INTRODUÇÃO}

O handebol é definido como um esporte olímpico de alta intensidade, que exige demandas aeróbicas e de força muscular esquelética. Classifica-se como um esporte coletivo, de caráter olímpico determinado pelo desempenho individual e interação coletiva entre os membros da equipe (OLIANO et al., 2017).

Contém elementos táticos de grande importância, como ações de corrida, arremesso, salto, contato e bloqueio (OLIANO et al., 2017). Essas movimentações são repetidas várias vezes durante o jogo, intercaladas por atividades intensas de baixa a moderada, o que adiciona um componente aeróbico considerável ao jogo. Esse padrão de atividade tem como consequência uma frequência cardíaca média de treinamento acima de 80\% da frequência cardíaca máxima (MADSEN et al., 2019).

Além disso, é um esporte de contato corporal muito exaustivo, caracterizado por habilidades motoras altamente desenvolvidas, como velocidade, poder explosivo, resistência e força. O desempenho da atleta no handebol feminino depende diretamente de diversos atributos fisiológicos, entre eles força e velocidade (LIDOR; ZIV, 2016). A pesquisa de Camacho-Cardenosa et al. (2018) demonstra que a composição corporal pode influenciar no desempenho do jogador em uma partida, tendo em vista que o tamanho da mão e a força de preensão de cada jogador tem influência direta no controle da bola, do passe e, consequentemente, do arremesso.

Fallahi e Jadidian (2011) caracterizam a força de preensão como uma importante ferramenta para agarrar e arremessar. Segundo esses autores, em esportes que exigem essas características, como o handebol, quanto maior a força de preensão, maior precisão terá o arremesso, uma vez que o movimento do arremesso é finalizado pelo punho e pelos dedos. Gonçalves e Santana (2013) referem que a avaliação da demanda metabólica em modalidades esportivas intermitentes torna-se necessária para compreender como o desempenho e o treinamento devem ser realizados. Nesse sentido, a avaliação da capacidade cardiorrespiratória nos esportes é uma prática muito comum para estabelecer níveis de condicionamento e poder melhorá-la (ZANELLA et al., 2015).

Neste estudo, teve-se como objetivo avaliar a força de preensão e a frequência cardíaca de treinamento em atletas amadoras do time de handebol da Universidade Federal de Santa Maria (UFSM), Santa Maria - RS.

\section{METODOLOGIA}

Este estudo é de caráter quantitativo transversal. Os sujeitos da pesquisa são atletas constituintes do time de handebol feminino amador adulto da Universidade Federal de Santa Maria, do município de Santa Maria - RS, que estavam em treinamento regular. Os critérios de inclusão foram: ser atleta do gênero feminino; não ter lesões musculares e ósseas no momento da avaliação; estar dentro da faixa etária de 18 a 27 anos; estar treinando a modalidade esportiva por, no mínimo, um ano; 
ter disponibilidade de horário; aceitar participar da pesquisa e assinar o Termo de Consentimento Livre e Esclarecido (TCLE). Definiu-se como critérios de exclusão: atletas com lesões musculares na data da avaliação; atletas com doenças cardíacas, neurológicas e ósseas que pudessem impossibilitar a realização do teste proposto; atletas que não estivessem realizando treinamento há mais de uma semana; participantes de outro tipo de esporte coletivo ou individual. O trabalho foi aprovado pelo Comitê de Ética e Pesquisa da Universidade Franciscana, sob o protocolo $n^{\circ}$ 04319118.3.0000.5306.

O time é composto por 22 atletas com média de idade de 19,8 anos. Mediante análise dos critérios de inclusão no estudo, 12 atletas estavam aptas para participar da pesquisa. Estas participaram do protocolo de avaliação, que foi composto por uma ficha de identificação em que constavam dados pessoais, dados antropométricos (IMC), idade, membro superior dominante, posição em quadra e tempo de prática da modalidade.

O índice de massa corporal (IMC) é um dos índices mais utilizados na área da composição corporal e é caracterizado como a divisão do peso pela altura elevada ao quadrado, no qual o peso corporal é expresso em $\mathrm{kg}$ e a altura em m² (BARBOSA, 2013). A avaliação da força de preensão foi realizada por meio do aparelho dinamômetro da marca ADInstruments, modelo MLT003/D. Durante a avaliação, a atleta estava sentada em uma cadeira com apoio de tronco, com cotovelo posicionado em $90^{\circ}$, utilizando uma luva antiderrapante. Foi solicitado que ela comprimisse o aparelho com máxima força durante três segundos para gerar um pico de força, processo realizado três vezes com ambos membros superiores com intervalo de um minuto entre as repetições (BIAZUS et al., 2020).

Por fim, foi efetuada a avaliação da frequência cardíaca (FC) das atletas ao longo de um treino com duração de duas horas. Para isso, foi utilizado um frequencímetro da marca Polar. A FC foi verificada em três momentos: dez minutos antes do treino, com a atleta em estado de repouso, após uma hora de treino e dez minutos com a atleta em estado de repouso novamente, após o fim do treino. Foi realizada a média dessas medições, o cálculo da frequência cardíaca máxima (FCmáx) e o cálculo dos valores médios da FC (\%) em relação à FCmáx das atletas (GHORAYEB et al., 2019).

Para a análise estatística, foi utilizada a estatística descritiva (média, desvio padrão e porcentagem), e os dados foram apresentados em gráficos e tabelas para facilitar a visualização e a compreensão.

\section{RESULTADOS}

O estudo foi realizado com 12 participantes do time amador de handebol feminino da UFSM de Santa Maria - RS. As atletas têm uma faixa etária de 19,8 $\pm 1,2$ anos, tempo de treinamento de $3,3 \pm 1,5$ anos. Todas possuem o membro superior direito dominante, não possuem uma posição fixa em quadra e treinam em média cinco vezes na semana.

Na tabela 1, constam as características gerais das atletas, como o perfil antropométrico (IMC) e média de frequência cardíaca de treinamento. A amostra é caracterizada por 12 atletas do gênero 
feminino, jovens, com índice de massa corpórea normal e que atingem um percentual de 79\% de sua frequência cardíaca máxima.

Tabela 1 - Características morfológicas e antropométricas das atletas.

\begin{tabular}{|cc}
\hline Variáveis & Característica das atletas \\
\hline Idade & $19,8 \pm 1,2$ \\
\hline IMC & $24,3 \pm 2,9$ \\
\hline FC de treinamento & $154,1 \pm 10,9$ \\
\hline FC máxima & $193,5 \pm 1,2$ \\
\hline FC (\%) & $79 \%$ \\
\hline Total de atletas & $\mathbf{1 2}$ \\
\hline
\end{tabular}

Fonte: Construção do autor.

No gráfico 1 e na tabela 2, são apresentados os valores de força de preensão dos membros superiores direito $(5,4 \pm 1,1 \mathrm{kgf})$ e esquerdo $(5,3 \pm 0,8 \mathrm{kgf})$ das atletas. Como pode ser observado, não existe diferença significativa entre as forças obtidas pelos membros direito e esquerdo.

Gráfico 1 - Força de preensão dos membros superiores direito e esquerdo quando comparados individualmente.

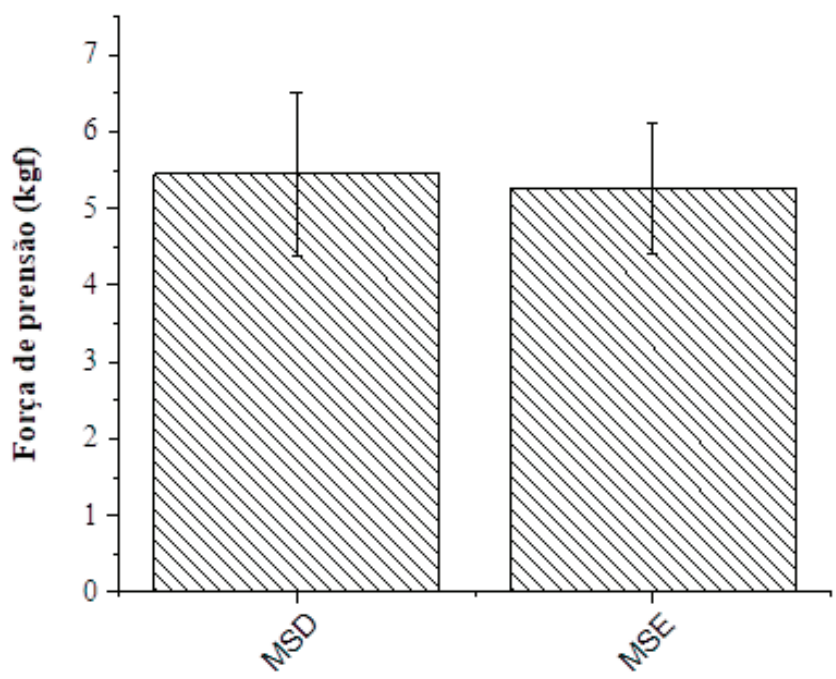

Fonte: construção do autor

Tabela 2 - Média da força de preensão entre os membros superiores.

\begin{tabular}{cccc}
\hline & N & Média & Desvio padrão \\
\hline MSD & 12 & 5,4 & 1,1 \\
MSE & 12 & 5,3 & 0,8 \\
\hline \multicolumn{4}{c}{ Fonte: construção do autor }
\end{tabular}

\section{DISCUSSÃO}

Nesta pesquisa, após analisada a variável força de preensão, ela se mostrou homogênea nas atletas quando comparadas individualmente. Esse resultado pode ser justificado pelo motivo de as 
atletas treinarem com ambas as mãos, tanto para trabalhos de passes com a bola como de arremessos a gol. Outro fator importante é que, por ser um time de handebol amador, a equipe optou por não designar para as atletas uma posição fixa em jogo, ou seja, dependendo da demanda exigida pelo jogo, são escolhidas as posições que as atletas irão entrar em quadra.

Pizzigalli et al. (2017), com uma amostra de 106 atletas de basquete do gênero feminino, com a faixa etária média de 19,4, analizaram a força de preensão das atletas, na qual elas foram divididas em subgrupos por idade (abaixo de 14 anos, abaixo de 16 anos, abaixo de 18 anos, abaixo de 20 e acima de 20 anos). Quando correlacionadas as atletas acima de 20 anos, também não foram encontradas diferenças estatísticas entre elas.

Outra pesquisa realizada com 19 atletas de judô do gênero feminino com uma média de 20,8 anos avaliou a força de preensão, demonstrando que as atletas com maior força de preensão têm um maior desempenho durante a partida (KONS et al., 2018).

Nas modalidades, como handebol, judô, voleibol, basquetebol, tênis e tênis de mesa, as capacidades física, mental, técnica e tática são fundamentais para o desempenho do atleta. Contudo, a força de preensão manual é uma qualidade física importante nesses esportes e, quando aliada aos aspectos morfológicos e funcionais das mãos, pode ser fundamental para o bom desempenho (FERNANDES; MARINS, 2011).

O handebol tem alta exigência do ponto de vista aeróbico. No estudo de Póvoas et al. (2017), foi observado que, em uma partida de sessenta (60) minutos, os jogadores percorreram em média $4 \mathrm{~km}$, alcançando uma intensidade média de $87 \%$ da frequência cardíaca, o que comprova que $90 \%$ da energia gasta em uma partida é de forma aeróbica. No presente estudo, encontrou-se uma média de frequência cardíaca máxima de treinamento de 79\%, o que está de acordo com o estudo de Póvoas et al., mesmo quando comparado a atletas homens, respeitando as diferenças fisiológicas.

Outro estudo relata também que, quando analisadas as demandas fisiológicas do jogo de handebol, a frequência cardíaca (FC) tem valores médios de $82 \%$ da FC máxima (FCmáx) individual em jogadores adultos (RAVIER; HASSENFRATZ; BOUZIGON, 2017). Barbero et al. (2014) realizaram um estudo semelhante em uma população masculina de um time de elite em que identificaram que a frequência cardíaca média durante um treinamento foi de $160 \pm 10 \mathrm{bpm}$, o que se refere a $82,5 \pm 4,7 \%$ da frequência cardíaca máxima dos jogadores.

Tendo em vista que a população estudada é do gênero feminino e atletas amadoras, a frequência média atingida por elas mostra que é necessário maior preparo no quesito aeróbico para que essas demandas tenham um alcance maior, aumentando também o desempenho em quadra das atletas (SILVA et al., 2015).

Para que se possa atingir o desempenho máximo da jogadora no handebol, é essencial usar o conhecimento de vários domínios relacionados a essa modalidade (velocidade, destreza) e ao esporte, incluindo a fisiologia do exercício e a medicina esportiva (MANCHADO et al., 2013). 
Há poucos estudos que abranjam a população de atletas femininas de handebol tanto no âmbito amador como no profissional, o que dificultou o enriquecimento do presente estudo. Assim, são necessárias mais pesquisas relacionadas a atletas amadoras do gênero feminino praticantes de handebol.

\section{CONCLUSÃO}

Neste estudo, foi avaliada a força de preensão das atletas e não foi encontrada uma diferença estatística entre os membros superiores direito e esquerdo e a frequência cardíaca de treinamento. Esse resultado demonstra uma homogeneidade no perfil das atletas, que permaneceu, na maior parte do tempo, entre $60 \%$ e $70 \%$ da FC de treinamento.

Com esta pesquisa, percebeu-se que a dinâmica utilizada pela equipe tanto para treinos como para competições levam a uma proximidade de força muscular de preensão para ambos os membros.

Como o handebol é um esporte coletivo e de rendimento, é de fundamental importância o papel do fisioterapeuta em uma equipe multidisciplinar esportiva, posto que a fisioterapia desportiva tem como domínios a prevenção, a reabilitação, os atendimentos emergenciais e o retorno à atividade do esporte.

\section{REFERÊNCIAS}

BARBERO, J. C. et al. Physical and physiological demands of elite team handball players. Int J Perform Anal Sport, v. 14, n. 3, p. 921-933, 2014.

BARBOSA, D. C. L. Indicadores antropométricos de risco cardiovascular em adultos. 2013. Disponível em: https://bit.ly/3ergUTB

BIAZUS, J. D. F. et al. Eficácia da terapia aquática em indivíduos portadores de fibromialgia. Ciências da Saúde: Campo Promissor em Pesquisa. Editora Atena, v. 9, cap. 5, p. 29, 2020.

CAMACHO-CARDENOSA, A. et al. Anthropometric and Physical Performance of Youth Handball Players: The Role of the Relative Age. Sports, v. 6, n 2, p. 47, 2018.

FALLAHI, A. A.; JADIDIAN, A. A. The effect of hand dimensions, hand shape and some anthropometric characteristics on handgrip strength in male grip athletes and non-athletes. Journal of human kinetics, v. 29, p. 151-159, 2011.

FERNANDES, A. D. A.; MARINS, J. C. B. Teste de força de preensão manual: análise metodológica e dados normativos em atletas. Fisioter. Mov., Curitiba, v. 24, n. 3, p. 567-578, 2011 
GONÇALVES, H. R.; SANTANA, W. C. Valores de frequência cardíaca de jogadores de futsal em situação de jogo. Pensar a Prática, v. 16, n. 1, p. 13-19, 2013.

GHORAYEB, N. et al. The Brazilian Society of Cardiology and Brazilian Society of Exercise and Sports Medicine Updated Guidelines for Sports and Exercise Cardiology. Arq. Bras. Cardiol. v. 112, n. 3, p. 326-368, 2019.

KONS, R. L. et al. Female Judo Athletes' Physical Test Performances Are Unrelated to Technical-Tactical Competition Skills. Perceptual and Motor Skills, v. 125, n. 4, p. 802-816, 2018.

LIDOR, R.; ZIV, G. Physical and Physiological Attributes of Female Team Handball Players - A Review. Women Sport Phys Act J, v. 20, n. 1, p. 23-38, 2016.

MADSEN, M. et al. Activity Profile, Heart Rate, Technical Involvement, and Perceived Intensity and Fun in U13 Male and Female Team Handball Players: Effect of Game Format. Sports, v. 7, n. 4, p. 90, 2019.

MANCHADO, C. et al. Performance factors in women's team handball: Physical and physiological aspects-a review. Journal of Strength and Conditioning Research, v. 27, n. 6, p. 1708-1719, 2013.

OLIANO, V. J. et al. Effect of FIFA 11+ in addition to conventional handball training on balance and isokinetic strength. Rev. bras. cineantropom. desempenho hum, v. 19, n. 4, p. 406-415, 2017.

PIZZIGALLI, L. et al. Hand grip strength and anthropometric characteristics in Italian female national basketball teams. J Sports Med Phys Fitness, v. 57, p. 521-528, 2017.

PÓVOAS, S. et al. Physical and Physiological Demands of Recreational Team Handball for Adult Untrained Men. BioMed research international, v. 2017, p. 1-10, 2017.

RAVIER, G.; HASSENFRATZ, C.; BOUZIGON, R. Reproducibility of heart rate and perceptual demands of game-based training drills in handball players. Rev. bras. cineantropom. desempenho hum., v. 19, n. 5, p. 515, 2017.

SILVA, A. A. et al. Análise do perfil, funções e habilidades do fisioterapeuta com atuação na área esportiva nas modalidades de futebol e voleibol no Brasil. Rev Bras Fisioter., v. 19, n. 3, p. 278-282, 2015.

ZANELLA, A. L. et al. Consumo máximo de oxigênio de jogadores de voleibol: comparação entre posições de jogo. Revista Brasileira de Prescrição e Fisiologia do Exercício, v. 9. n. 54. p. 404-409, 2015. 
